\title{
Implicaciones bioéticas en el «efecto de contagio» del suicidio
}

\section{Bioethical implications in the «contagion effect» of suicide}

\author{
Érika Benítez Camacho*
}

https://doi.org/10.36105/mye.2022v33n1.04

\section{Resumen}

El suicidio es una problemática grave de salud pública en México y en el mundo, el cual, de acuerdo con la Organización Mundial de la Salud, cobra la vida de alrededor de 800 mil personas al año. Pese a que la muerte autoprovocada tiene origen multifactorial, es de suma relevancia el reconocimiento de los diversos factores de riesgo y la corresponsabilidad social que se tiene en los mismos. En particular, el «efecto de contagio» del suicidio es un fenómeno que aún falta por explorar, no sólo desde las perspectivas de las ciencias de la salud o de la comunicación, sino también desde la bioética. Es por ello que en este artículo no sólo se describe el "efecto de contagio» del suicidio, sino que se analizan las implicaciones bioéticas de la influencia que tiene en la muerte autoprovocada la exposición a noticias divulgadas por los medios de comunicación que no siguen los lineamientos éticos de la Organización Mundial de la Salud.

Palabras clave: suicidio, «efecto de contagio» del suicidio, salud mental, medios de comunicación, vulnerabilidad social.

\footnotetext{
* Universidad Anáhuac México, Facultad de Psicología. México. Correo electrónico: ebenitez@anahuac.mx https://orcid.org/0000-0002-8522-7145

Recepción: 15 de agosto de 2021. Aceptación: 12 de septiembre de 2021.
}

Medicina y Ética - Enero-Marzo 2022 - Vol. 33 - Núm. 1 


\section{Introducción}

La conducta suicida, y en particular la muerte autoprovocada, se ha incrementado en diversos países del mundo. México no ha sido la excepción. De acuerdo con la Organización Mundial de la Salud (1, 2), cada año alrededor de 800 mil personas se suicidan, lo que equivale a la muerte autoinfligida de una persona cada 40 segundos (3); sin embargo, existe preocupación sobre un aumento en las cifras asociadas a los retos y adversidades que se han tenido que enfrentar en los últimos tiempos en las áreas sanitarias, económicas y sociales.

La muerte autoprovocada es multifactorial. Su etiología se vincula con trastornos mentales, trastornos de la personalidad, crisis agudas de vida, infelicidad profunda, pérdida del sentido de la vida y ausencia de un proyecto vital. Sin embargo, es importante considerar también la influencia de elementos que se suman a condiciones críticas y que constituyen factores de riesgo, tales como la exposición a contenidos de medios masivos de comunicación que promueven la violencia y que, en particular en relación con la conducta suicida, no siguen las pautas éticas de la Organización Mundial de la Salud. En este artículo se escribirá sobre lo que se ha denominado el «efecto de contagio» del suicidio y, por ende, la vinculación entre la conducta suicida y los medios masivos de comunicación, así como las implicaciones bioéticas que entrelazan ambos temas.

\section{Medios de comunicación y exposición a la violencia}

Los medios de comunicación masivos influyen significativamente en el desarrollo de la cultura (4). Cuando los contenidos mediáticos son violentos, el impacto mental en las personas, sobre todo en niños y adolescentes, es relevante (5), pudiendo incluso generar aislamiento social, conductas destructivas, ciberludopatía, adicción a los medios, problemas de salud mental, trastornos de conducta 
de tipo antisocial y desensibilización a la violencia, entre otros (6-12). Ante esta exposición mediática, los jóvenes son los que parecen más afectados; sin embargo, también hay evidencia del impacto adverso que tienen las noticias violentas en todo tipo de personas, indistintamente de la edad, del género y de la cultura, pues el contenido mediático muy cercano influye en la percepción que tengan de la violencia $(13,14)$.

Por tanto, es fundamental que los padres monitoreen los contenidos de los medios de comunicación que ven o escuchan sus hijos niños o adolescentes, pues así se podrá lograr un factor protector sobre su desarrollo físico, emocional, académico y social (15). Asimismo, es relevante el desarrollo de estrategias mediáticas en contra de la violencia, de manera que influyan en la diminución de la misma $(16,17)$.

\section{Medios de comunicación y «efecto de contagio» en el suicidio}

Sobre el tema del suicidio, se ha estudiado el «efecto de contagio» que pueden producir los medios de comunicación, los cuales tienen un rol trascendental en la vida actual de la población, en particular de los adolescentes, quienes consideran que la tecnología y el uso de las redes sociales son parte fundamental de su vida (18).

La primera alusión al fenómeno de contagio del suicidio se hace con el llamado «efecto Werther», denominado así porque tras la publicación del libro Las penas del joven Werther, de Johann Wolfgang von Goethe (publicado en 1774 y en donde el protagonista se suicida), se observó un incremento en los suicidios de los jóvenes en circunstancias y vestimenta similar a las del protagonista de la novela. Posteriormente, el sociólogo David P. Phillips investigaría este fenómeno al observar el aumento de suicidios después de que se publicara una nota sobre el suicidio en periódicos de Estados Unidos, particularmente en el New York Times. Sus investigacio-

Medicina y Ética - Enero-Marzo 2022 - Vol. 33 - Núm. 1 
nes concluyeron que la cobertura por parte de los medios de comunicación sobre el suicidio incrementaba la tasa de suicidios en la población en la que se llevaba a cabo dicha cobertura $(19,20)$. Otros autores (21) corroboraron esto al identificar que el suicidio en adolescentes en Estados Unidos desde 1988 a 1996 se presentaba en grupos, y no sólo de manera aislada, cuando los periódicos reportaban los suicidios en la primera página, describían el método del suicidio y colocaban encabezados con la palabra «suicidio». Por el contrario, por ejemplo, en Viena se evidenció que cuando la información que se daba por parte de los medios de comunicación sobre notas de suicidio ocurridos en el Metro era más breve y menos dramática, los suicidios e intentos de suicidio posteriores disminuían considerablemente (22).

Japón es uno de los países que más ha estudiado si la cobertura por parte de los medios de comunicación sobre el suicidio influye en la repetición de la conducta suicida. ${ }^{1}$ Han concluido que las imágenes sobre el suicidio influyen de manera importante para que personas vulnerables psicológicamente intenten suicidarse, utilizando incluso métodos que no son usuales para la cultura (23). En el mismo país, las investigaciones realizadas sobre el impacto de la difusión del suicidio por medio de periódicos señalan que si la noticia sobre el suicidio o intento de suicidio se coloca en primera página, tiene un impacto cuatro veces mayor en las personas que si se coloca en otra parte del periódico. Dicho impacto se incrementa si lo hace también el tiempo de exposición a la noticia, que en Japón es de uno a tres días (es decir, la noticia sobre un suicidio consumado o intento de suicidio se expone en promedio de uno a tres días en los periódicos). Si los medios de comunicación no siguen las recomendaciones que se han hecho en ese país y de manera internacional sobre la difusión del suicidio, el efecto de contagio o copy-cat se incrementa de manera significativa (24).

En China, el efecto de contagio se ha estudiado mediante el análisis de la propagación del método del suicidio, el cual puede trascender las fronteras, tal como ocurrió entre los años de 1998 a 
Implicaciones bioéticas en el «efecto de contagio» del suicidio

2005 con el salto de altura (como medio para suicidarse), que se extendió a Taiwán, Japón, Corea y Singapur. Sin embargo, estas investigaciones no sólo se centran en la huella que deja en la población la difusión del método de suicidio por parte de los medios de comunicación, sino también de las motivaciones del mismo, tales como el divorcio o las crisis financieras, las cuales son un factor de riesgo para la conducta suicida en China y en el mundo. La incidencia del efecto de contagio es mayor en zonas urbanas que en zonas rurales (25).

En la cultura occidental también se han encontrado evidencias del efecto de contagio del suicidio y su relación con los medios de comunicación. Un ejemplo de esto fue el libro Final Exit, que ofrecía una guía para el suicidio de los enfermos terminales; el método que sugería era la asfixia. Durante el año de publicación de este libro (1991), los suicidios por este método se incrementaron un 313\% en la ciudad de Nueva York, encontrándose en el 27\% de los suicidios -en el lugar del fallecimiento- un ejemplar de este libro (26).

Otro ejemplo es la serie de televisión estadounidense 13 razones por qué, en donde se muestra el suicidio de una joven y las motivaciones aparentes que la llevaron a cometer ese acto. Una investigación realizada en Estados Unidos señaló que desde el estreno de la serie el 31 de marzo de 2017 hasta el 18 de abril del mismo año, se observó un incremento de búsquedas en internet relacionadas con el suicidio. En 12 de los 19 días que se estudiaron, las consultas sobre el suicidio fueron significativamente más altas, oscilando entre un $15 \%$ (al 15 de abril de 2017) y un 44\% (al 18 de abril de 2017). $\mathrm{El}$ incremento en las búsquedas específicas sobre «cómo cometer suicidio» fue del $26 \%$; «cometer suicidio» un 18\%, y «cómo matarte a ti mismo», un $9 \%$. Las consultas en internet sobre la prevención del suicidio y las líneas telefónicas para recibir ayuda también aumentaron («suicidio adolescente» incrementó un 34\%; «prevención del suicidio», 23\%; «números de las líneas directas del suicidio» o «suicide botline number», $21 \%, \mathrm{y}$ «líneas directas del suicidio» $\mathrm{o}$ «suicide botline», 12\%) (27). Un estudio identificó que la tasa de suicidios en

Medicina y Ética - Enero-Marzo 2022 - Vol. 33 - Núm. 1 
jóvenes varones de entre 10 y 17 años en Estados Unidos creció un $28.9 \%$ el mes siguiente al estreno de la primera temporada de esta serie de televisión y se mantuvo elevada los dos meses subsecuentes (28). ${ }^{2}$

$\mathrm{El}$ «efecto de contagio» se evidencia en una investigación canadiense del año 2018, que establece la asociación entre los factores dañinos y protectores de los reportajes en los medios de comunicación sobre el suicidio. Se ha identificado que algunos de los elementos que aumentan el suicidio en la semana siguiente a la publicación de una nota de suicidio o intento de suicidio son: afirmación de que el suicidio era inevitable (1.97 veces); colocar el método suicida en el encabezado (1.41 veces); si el método de suicidio es ahorcamiento o asfixia (1.72 veces); si el método es salto de un edificio (1.7 veces); hablar de un pacto suicida (1.63 veces); si el método suicida es por arma de fuego (1.3 veces); si el suicidio es de una persona famosa (1.3 veces), y si el suicidio es de un adulto mayor (1.25 veces) (29).

$\mathrm{Al}$ contrario, los factores de protección para el suicidio son: políticas públicas para evitar el suicidio; señalamiento de las características de personalidad desfavorables de la persona fallecida; si el método de suicidio fue arrojarse a las vías del tren o por medio de cortes o apuñalamiento, o si se trataba de una persona que primero fue homicida y luego suicida (29).

\section{Factores que incrementan el «efecto de contagio» en el suicidio}

El efecto de contagio del suicidio se basa en la asociación existente entre los siguientes elementos (30): a) una conducta por contagios masivos relacionados en el tiempo que superan las barreras geográficas y que tienen en común las noticias que se difunden sobre el suicidio de una celebridad (mass clusters); b) por contagios puntuales o relacionados en el espacio-tiempo (point/space-time clusters) -por 
ejemplo, los ocurridos en prisiones, instituciones, hospitales o escuelas-, o los que ocurren por imitación de un suicidio (echo cluster), generalmente asociados a la cobertura mediática del mismo.

La influencia del suicidio expuesto en los medios de comunicación parece ser mayor cuando la noticia es sobre una persona famosa, pues la cobertura es repetitiva durante días y más específica sobre las circunstancias alrededor del suicidio, el aparente motivo y el método mismo (31-33). Pero también tiene un efecto mayor debido a que las personas crean relaciones parasociales con los famosos; es decir, que, aunque el público no conozca personalmente a la celebridad, establece una relación afectiva con la misma, se identifica con ella e incluso siente conocerla (34). El impacto aumenta si la persona famosa y el público son de la misma nacionalidad (incrementa el grado de identificación personal) y si existe un ambiente suicidogénico en la población (por ejemplo, condiciones de vida adversas) (35).

En algunos casos, los medios de comunicación exponen de manera sensacionalista y con un uso inapropiado del lenguaje la noticia sobre el suicidio de una celebridad; dan a entender que el suicidio era el camino lógico posible y no mencionan los probables trastornos mentales subyacentes al suicidio. La mayoría de las veces las noticias se acompañan de imágenes sobre la muerte o el cuerpo fallecido (36), lo cual, aunado a lo que ya se ha mencionado, incrementa hasta 14 veces la posibilidad del «efecto de contagio» sobre el suicidio (37). Éste se concentra en las siguientes dos semanas a la cobertura de la muerte de la persona famosa, pero puede continuar hasta cuatro semanas después. En cambio, si los medios de comunicación no difunden abiertamente la noticia sobre el suicidio, el «efecto de contagio» permanece sólo una semana (38).

Ejemplo de cómo la difusión del suicidio de una persona famosa puede incrementar la tasa de suicidios es Corea. Los investigadores (32) revisaron de manera sistemática la noticia sobre el suicidio de una famosa cantante coreana, ${ }^{3}$ la prevalencia de casos de intento 
de suicidio o suicidio consumado y las motivaciones de dichos intentos de suicidio con entrevistas directas de las personas que lo cometieron, y llegaron a la conclusión de que la tasa de intentos de suicidio y suicidios consumados se incrementa en las cuatro semanas posteriores a la difusión de la noticia sobre el suicidio de un personaje famoso, y que dicha tasa está relacionada con el efecto de imitación de la conducta, la identificación del personaje famoso por medio de la edad, de la motivación asociada y del método empleado. ${ }^{4}$

Por otro lado, el uso de internet y de las redes sociales pueden ser medios para la prevención del suicidio, o bien para su favorecimiento. Adolescentes y adultos jóvenes pueden verse altamente influenciados por el contenido a favor del suicidio que se presenta en algunas redes sociales, el cual se encuentra fácilmente en las búsquedas realizadas en internet (39). Incluso existen sitios específicos que inducen al suicidio de manera explícita, y otros que no lo hacen abiertamente en el título, pero que tienen un contenido (verbal, en imágenes, videos, películas o bandas musicales) que orienta al suicidio (40).

En suma, se ha encontrado que los medios de comunicación influyen en la repetición de la conducta suicida, pues la cobertura que hacen de las noticias en periódicos, televisión e internet, se enfoca en mostrar los detalles del método suicida, pero no exponen con responsabilidad las causas de la muerte autoprovocada. Generalmente, atribuyen el acto suicida a una crisis cualquiera $\mathrm{o}$ a fracasos personales, pero no indican lo complejo que es la conducta suicida, los trastornos mentales que pueden existir de manera subyacente y la vulnerabilidad que pueden presentar algunas personas $(41,42)$.

Desde esta perspectiva, se recomienda ser cuidadoso con la forma en que se difunden las notas de suicidio (43-45). Es fundamental que los medios de comunicación se guíen por medio de lineamientos éticos que promuevan la información veraz y la salud mental, así como que minimicen el daño que su contenido puede generar, 
Implicaciones bioéticas en el «efecto de contagio» del suicidio

tanto a los protagonistas de una nota, como al público en general $(4,46,47)$. Los medios de comunicación deben transmitir información de acuerdo con los lineamientos de la Organización Mundial de la Salud (48-50).

\section{Efecto protector de los medios de comunicación en la conducta suicida}

La cobertura responsable de los medios de comunicación sobre el suicidio puede tener un efecto preventivo en el desarrollo de la violencia y la conducta suicida (51). Este efecto, conocido como «efecto Papageno», ${ }^{5}$ consiste en mostrar al público que existen maneras más efectivas y positivas de enfrentar las crisis emocionales sin la necesidad de recurrir a la conducta suicida (52). Puede tener consecuencias favorables cuando los familiares de la persona que se ha suicidado son entrevistados por los medios de comunicación de manera ética, con el propósito de evitar futuras conductas suicidas (53); cuando los medios de comunicación ayudan a disminuir el estigma y los estereotipos que se tienen sobre los trastornos mentales (54), y cuando difunden información sobre la recuperación que pueden lograr las personas que han intentado suicidarse y que han recibido atención médica y psicológica oportuna (55).

Como ya se ha mencionado, la Organización Mundial de la Salud, la Organización Panamericana de la Salud y el Fondo de las Naciones Unidas para la Infancia han emitido recomendaciones a los medios de comunicación sobre la forma de transmitir notas sobre la conducta suicida $(19,48,56-59)$, y han proporcionado guías a los cineastas para que puedan manejar de manera ética este tema en películas y series de televisión. ${ }^{6}$ Algunas de las recomendaciones realizadas son:

a) La noticia sobre el suicidio consumado o intento de suicidio no debe aparecer ni en la primera página ni en la última, que sería la inicial para los que comienzan a leer el periódico al revés.

Medicina y Ética - Enero-Marzo 2022 - Vol. 33 - Núm. 1 
b) No deben aparecer fotos del fallecido.

c) No se debe describir detalladamente el método utilizado, pues sirve a otros de referencia si están indecisos respecto a cuál medio utilizar para acabar con su vida.

d) No deben ofrecerse explicaciones simples ni únicas del suicidio, pues este fenómeno es una conducta compleja que responde a causas biológicas, psicológicas y sociales.

e) No debe equipararse el suicidio con atributos valiosos del carácter ni valores morales dignos de imitar, como la valentía, la lealtad, el amor, la dignidad, el honor o el altruismo.

f) No deben enfatizarse únicamente los aspectos positivos de la personalidad del difunto, sino también los posibles factores que facilitaron el suicidio, tales como padecer alguna enfermedad mental o el uso de sustancias.

g) No debe aparecer la palabra suicidio como sinónimo de éxito, salida, opción o solución.

h) No se debe dar a entender que el suicidio es una forma de resolver las dificultades de la vida.

Igualmente, se sugiere no mencionar el nombre ni las características de la persona que se suicidó y no hacer referencias a foros de internet sobre el suicidio, a pactos sobre el suicidio o de lugares en donde se localizan un mayor número de suicidios (detonan puntos de riesgo). Se debe evitar mencionar suicidios que están cerca en tiempo o espacio y, al contrario, se deben destacar factores de prevención, los cuales son escasamente colocados en las notas sobre la muerte autoprovocada (60).

Asimismo, se recomienda proporcionar información sobre los centros de atención en donde una persona con ideación suicida puede solicitar ayuda; destacar las alternativas favorables que existen frente a las crisis; difundir indicadores de riesgo y señales de advertencia para el suicidio, y enviar mensajes de solidaridad a los sobrevivientes del suicidio (61).

Estas recomendaciones aplican también a las plataformas de internet y redes sociales que usan los jóvenes, pues algunos las con- 
Implicaciones bioéticas en el «efecto de contagio» del suicidio

sultan como forma de socialización o en busca de información, pero otros (en el caso de aquellos vulnerables emocionalmente o con un trastorno mental), se acercan a ellas para obtener apoyo (62).

Una muestra de la falta de seguimiento a las recomendaciones de la Organización Mundial de la Salud fue el tratamiento que en el 2018 se les dio a los suicidios de dos personajes famosos: la diseñadora Kate Spade y el chef Anthony Bourdain. En ambos casos, las notas de sus suicidios fueron difundidas por medios de comunicación de todo el mundo y replicadas varias semanas después de los eventos.

En el caso de Kate Spade ${ }^{7}$ se mostraron imágenes y videos de la policía retirando el cadáver de la casa de la diseñadora; dieron a conocer la nota suicida que se encontró al lado de su cuerpo; exhibieron declaraciones de la hermana y del esposo de Spade, y detallaron cómo fue que la diseñadora se suicidó, qué método utilizó e incluso cómo y por quién fue encontrado su cuerpo. Algunos medios de comunicación mencionaron entrevistas en las que se indicaba que Kate sufría depresión y ansiedad desde hacía más de 5 años, para lo cual recibió tratamiento en un inicio. Aparentemente, el tratamiento fue abandonado posteriormente por un probable temor a que su marca se viera perjudicada $(63,64)$. Sólo algunos medios de comunicación incluían en sus notas una liga para obtener ayuda en caso de ideación suicida.

La difusión del suicidio de la diseñadora dio pie a múltiples reacciones por parte de los usuarios de redes sociales. Algunos comentaban sobre lo difícil que es padecer enfermedades mentales y lo complicado que es sobrevivir al suicidio de un familiar; otros criticaban la decisión de suicidarse y otros más, por el contrario, decían que el suicidio de Kate fue una buena decisión, pues de esa manera estaría salvando su empresa.

El suicidio del famoso chef Anthony Bourdain, ${ }^{8}$ ocurrido el 8 de junio del 2018 por ahorcamiento en el hotel Le Chambard de Kaysersberg, Francia, fue también una notica reportada por los

Medicina y Ética - Enero-Marzo 2022 - Vol. 33 - Núm. 1

https://doi.org/10.36105/mye.2022v33n1.04 
medios de comunicación alrededor del mundo. En la radio, televisión, internet y redes sociales se informó durante más de un mes sobre la muerte de Bourdain y la información derivada de la misma. Algunos medios fueron explícitos, al indicar que el chef murió por ahorcamiento y detallar el método utilizado. Otras noticias hicieron alusión a la depresión crónica que padecía Bourdain y a las adicciones con las que había luchado en el pasado. Incluso enfatizaron declaraciones que el chef había hecho en el 2017, en las que expresaba el arrepentimiento que sentía respecto del daño que habían provocado estas adicciones a los demás. Sólo algunos medios de comunicación hicieron alusión a los probables eventos desencadenantes sobre el suicidio, tales como la depresión, la crisis personal y económica (66).

Como se ha mencionado antes, el que los medios de comunicación sigan las recomendaciones de la Organización Mundial de la Salud y difundan de manera ética y responsable las noticias sobre el suicidio, ayuda a informar responsablemente a la población sobre las enfermedades mentales, con la consecuente disminución del estigma social de las mismas, así como a prevenir la conducta suicida y a disminuir los factores interpersonales de riesgo que afectan especialmente a los jóvenes $(67,68)$.

\section{Implicaciones bioéticas en el «efecto de contagio» del suicidio}

El «efecto de contagio» del suicidio debe ser analizado no sólo desde las áreas de salud mental y comunicación, sino también desde la perspectiva bioética, pues se debe partir de la premisa de que existen diversos factores de riesgo para el suicidio, entre ellos, la vulnerabilidad. Ésta es un principio propuesto en la Declaración de Barcelona de 1998. Es una dimensión antropológica que remite a la fragilidad y susceptibilidad de sufrir daños asociados a la propia 
Implicaciones bioéticas en el «efecto de contagio» del suicidio

condición humana (69). Sin embargo, también existe la vulnerabilidad social, marcador de desventaja, que hace referencia a grupos poblacionales marginados que tienen acceso limitado a la satisfacción de sus necesidades básicas y a la atención en su salud.

Si bien es cierto que no es lo mismo ser vulnerable que haber sido vulnerado, se debe reconocer que las personas con conducta suicida generalmente han sido vulneradas y que además presentan vulnerabilidad social, entendiendo este concepto como la vulnerabilidad antropológica intensificada por factores socioambientales que colocan a una persona o grupo en estado de indefensión, y que puede ser consecuencia de vivir en condiciones resultantes de marcada desventaja social $(70,71)$. En el caso de las personas con padecimientos psiquiátricos o psicológicos, o bien, con crisis de vida, la dificultad para tener acceso a los servicios de salud es, sin duda, una desventaja que los coloca en vulnerabilidad social. La alteración emocional por sí misma, la confusión y la duda existencial, así como probablemente la falta de una red de apoyo social, interfiere con la manera en que se interpretan los mensajes que se reciben del exterior, tales como los manifestados en los medios de comunicación.

En este sentido, se debe considerar el apego a las guías éticas de la Organización Mundial de la Salud sobre notas de suicidio en los medios de comunicación. Una de las recomendaciones bioéticas sería proporcionar guías claras y capacitación a los medios de comunicación sobre la transmisión de noticias relacionadas con el suicidio. Otras, promover la orientación hacia la verdad y bien universales, favorecer -mediante la familia y la sociedad- la consolidación de una identidad sólida, e impulsar la conformación de vínculos afectivos entre las personas para que éstas puedan formar una comunidad y sentirse parte efectiva de ella.

El suicidio también opera contra el principio de sociabilidadsubsidiariedad, el cual compromete a los integrantes de una comunidad a colaborar en la obtención del bien de todos, sobre todo si

Medicina y Ética - Enero-Marzo 2022 - Vol. 33 - Núm. 1

https://doi.org/10.36105/mye.2022v33n1.04 
ese bien se refiere a la promoción de la vida y al mantenimiento o procuración de la salud (72). Por ende, cuando se pierde a alguien de la comunidad, ésta debe cuestionarse qué hizo para que esa persona se suicidara y qué debe hacer para prevenir el suicidio.

Esto involucraría el análisis del «efecto de contagio» del suicidio por imitación e impacto, al conocer la nota del suicidio de otra persona e identificarse en algunos aspectos con ésta. Asimismo, considerar el sufrimiento de los familiares de una persona suicida, que se traduce en duelos complicados, desarrollo de enfermedades físicas y mentales y propios intentos suicidas $(73,74)$, de manera que el impacto desfavorable del «efecto de contagio» del suicidio no sólo afecta a una la persona que ejecuta el acto, sino a su familia y a otros miembros de la sociedad.

En el análisis bioético debiera considerar también la subsidiariedad, que no sólo compromete el respeto al bien que alguien pueda procurarse a sí mismo o al que alguien más procure a otro, sino a que en forma solidaria se apoye a aquellos que no pueden ayudarse a sí mismos o que no están en posibilidades de buscar la ayuda que requieren (75). Éste sería el caso de una persona que se encuentra desesperanzada, con sentido de vida disminuido, vacío existencial y/o crisis emocionales, y de aquellos que padecen algún trastorno mental y/o de la personalidad. Por subsidiariedad, se debiera proporcionar ayuda a quienes no la pueden alcanzar por sí mismos, pero también a quienes tengan mayor necesidad.

Finalmente, es preciso enfatizar el respeto que debe prevalecer a la dignidad e integridad de las personas humanas, así como la búsqueda de la construcción de una sociedad más empática y solidaria, promotora del bien común.

\section{Conclusiones}

El suicidio es una problemática de salud pública que se ha incrementado de manera significativa en México y en el mundo. Dado 
Implicaciones bioéticas en el «efecto de contagio» del suicidio

su origen multifactorial es relevante comprender los factores de riesgo y de protección asociados al mismo. Dentro de los factores de riesgo, cobra particular significancia el análisis del «efecto de contagio» del suicidio como un elemento de influencia desfavorable para las personas con vulnerabilidad social, como generalmente son aquellos que padecen algún trastorno mental, o que atraviesan por confusión, desesperanza o crisis de vida. El suicidio opera en contra de la sociabilidad y la subsidiariedad, rompe el tejido social y afecta a la comunidad; por tanto, es indispensable el apego a los lineamientos éticos de la Organización Mundial de la Salud respecto de la muerte autoprovocada, así como la promoción del respeto a la dignidad e integridad de la persona humana.

\section{Notas bibliográficas}

1 En Japón, al efecto de contagio sobre el suicidio se le denomina «efecto Yukiko», en alusión a una estrella de rock japonesa que se suicidó y que generó gran cobertura mediática; posteriormente se reportaron numerosos suicidios de adolescentes (23).

2 Debido a la controversia generada y a las recomendaciones de expertos de asociaciones como la American Foundation for Suicide Prevention, American Association of Suicidology y American School Counselor Association, el creador y los productores de la serie de televisión decidieron en verano del año 2019 editar la secuencia en la que la protagonista se quita la vida en la primera temporada, de manera que la escena de suicidio no se muestre explícitamente.

${ }^{3}$ El caso analizado fue del suicidio de Ivy Li, una famosa cantante coreana de 24 años, que provocó su muerte mediante la intoxicación de monóxido de carbono dentro de su coche. La historia sobre su suicidio apareció en primera plana y en los encabezados de los periódicos en tono sensacionalista. La noticia se siguió difundiendo con intensidad tres días después y todavía se la mencionaba en los medios de comunicación dos semanas más tarde. La nota detallaba el método del suicidio, las declaraciones de los padres y el aparente motivo, que era un «amor frustrado». Durante las cuatro semanas posteriores a la cobertura periodística del suicidio de Ivy Ly se presentaron 108 intentos de suicidio y suicidios consumados en hospitales (32).

${ }^{4}$ Por ejemplo, en las entrevistas directas realizadas a los sobrevivientes de intentos de suicidio por imitación de la cantante coreana Ivy Lee, los entrevistados mencionaron que habían intentado suicidarse porque eran «fans» de la cantante

Medicina y Ética - Enero-Marzo 2022 - Vol. 33 - Núm. 1 


\section{E. Benítez Camacho}

y porque, como ella, también habían sufrido en algún momento una decepción amorosa (32).

${ }^{5}$ Este efecto toma su nombre del personaje homónimo de la «Flauta mágica» de Mozart, que fue convencido de no suicidarse después de que tres niños le ayudaran a identificar otras alternativas para enfrentar la vida (52).

${ }^{6}$ El documento Prevención del suicidio: un recurso para los profesionales de los medios de comunicación, de la Organización Mundial de la Salud, se puede consultar en el siguiente enlace: https://www.who.int/mental_health/suicide-prevention/resource_booklet_2017/en/Las recomendaciones de la Organización Mundial de la Salud y la Organización Panamericana de la Salud.

${ }^{7}$ Kate Spade fue una diseñadora famosa de bolsas, carteras y otros accesorios, así como de línea de cama y regalos, entre otros productos. Su marca contaba con más de 315 tiendas alrededor del mundo. Se suicidó por ahorcamiento el martes 5 de junio del 2018, a los 55 años, en su departamento en Park Avenue, Nueva York. Su cuerpo fue encontrado por el ama de llaves, quien hizo declaraciones detalladas sobre la escena del suicidio. Kate dejó una nota suicida para su hija de 13 años, la cual fue filtrada a los medios de comunicación $(63,64)$.

${ }^{8}$ Anthony Michael Bourdain nació el 25 de junio de 1956 en la ciudad de Nueva York, Estados Unidos. Fue un reconocido chef, escritor de diversos libros y personalidad de la televisión, que tuvo gran éxito popularizando la cultura de la comida (65).

\section{Referencias bibliográficas}

1. Organización Mundial de la Salud. Suicidio. Datos y cifras. (Consultado el 28 de agosto de 2020). 2019. Disponible en: https://www.who.int/es/news-room/factsheets/detail/suicide

2. Organización Mundial de la Salud. World Health Statistics data visualizations dashboard. (Consultado el 28 de agosto de 2020). 2019. Disponible en: http:// apps.who.int/gho/data/node.sdg.3-4-viz-2?lang=en

3. Organización Mundial de la Salud. Cada 40 segundos se suicida una persona. (Consultado el 31 de agosto de 2020). 2019. Disponible en: https://www.who.int/ es/news-room/detail/09-09-2019-suicide-one-person-dies-every-40-seconds

4. Campbell R, Martin C, Fabos B. Media and culture. Mass communication in a digital age. Boston: Bedford/St. Martin's; 2015.

5. Esteinou J. Los medios de información colectivos y la producción de la violencia social. En Rebelil MA, Gómez DG (Coords.). Violencia mediática e interactiva, México: Trillas. 2009; 100-124.

6. Groebel J. La violencia en los medios. Estudio Global de la UNESCO. En: Proyecto principal de educación en América Latina y el Caribe. Santiago de Chile: Publicaciones OREALC; 1999. 
Implicaciones bioéticas en el «efecto de contagio» del suicidio

7. Leiner $\mathrm{M}$, Puertas $\mathrm{H}$, Caratachea R, Ávila $\mathrm{C}$, Atluru A, Briones D, et al. Children's mental health and collective violence: A binational study on the United States-México border. Rev Panam Salud Pública. 2012; 31(5), 411-416. https:// doi.org/10.1590/S1020-49892012000500009

8. Leiner M, Villanos MT, Puertas H, Peinado J, Ávila C, Dwivedi A. The emotional and behavioral problems of children exposed to poverty and/or collective violence in communities at the Mexico-United States border: A comparative study. Salud Mental. 2015; 38(2): 95-102. https://doi.org/10.17711/SM.0185-3325.2015.013

9. Martínez-Lanz, P. Internet y violencia: factores de riesgo en la salud mental de los jóvenes. En Rebelil MA, Gómez DG (Coords.), Violencia mediática e interactiva. Trillas, México. 2009; 166-180.

10. Twenge JM, Joiner TE, Rogers ML, Martin GN. Increases in depressive symptoms, suicide-related outcomes, and suicide rates among us adolescents after 2010 and links to increased new media screen time. Clinical Psychological Science. 2018; 6(1), 3-17. https://doi.org/10.1177/2167702617723376

11. Lee P. Violence. En L Wilkins \& CG Christians (Eds.), The Handbook of mass media ethics. Nueva York, Routledge. 2009; 162-176.

12. Claudia ME. Study on the effects of prolonged mass media consumption on the emotional state of the receptors. Journal of Educational Sciences \& Psychology. 2016; 6(1): 47-55.

13. Hawton K, Williams K. Influences of the media on suicide. BMJ. 2002; 325 : 1374-1375. https://doi.org/10.1136/bmj.325.7377.1374

14. Jesmin SS, Amin I. Impact of the mass media in changing attitudes towards violence against women in Bangladesh: Findings from a national survey. Journal of Family Violence. 2017; 32(5): 525-534.

https://doi.org/10.1007/s10896-016-9837-8

15. Gentile DA, Reimer RA, Nathanson AI, Walsh DA, Eisenmann JC. Protective effects of parental monitoring of children's media use: A prospective study. JAMA Pediatrics. 2014; 168(5): 479-484.

https://doi.org/10.1001/jamapediatrics.2014.146

16. Clark CJ, Spencer RA, Shrestha B, Ferguson G, Oakes JM, Gupta J. Evaluating a multicomponent social behaviour change communication strategy to reduce intimate partner violence among married couples: Study protocol for a cluster randomized trial in Nepal. BMC Public Health. 2017; 17(1): 75.

https://doi.org/10.1186/s12889-016-3909-9

17. Jashinsky JM, Magnusson B, Hanson C, Barnes M. Media agenda setting regarding gun violence before and after a Mass shooting. Frontiers in Public Health. 2017; 4: 291. https://doi.org/10.3389/fpubh.2016.00291

18. Barrera J, Gómez-López MT, Suárez E, Velásquez N, Guzmán Y, García V. Cobertura periodística sobre el suicidio: ¿Habría riesgo de causar efectos negativos en personas susceptibles? Pers. Bioét. 2017; 21(1): 151-160.

https://doi.org/10.5294/pebi.2017.21.1.10

19. Phillips DP. The influence of suggestion on suicide: Substantive and theorical

Medicina y Ética - Enero-Marzo 2022 - Vol. 33 - Núm. 1 
implications of the Werther Effect. American Sociological Review. 1974; 39: 340359. https://doi.org/10.2307/2094294

20. Marsden P. Operationalising memetics-suicide the werther effect and the work of David P. Phillips. 2018. Disponible en: http://pespmc1.vub.ac.be/Conf/MemePap/Marsden.html

21. Gould MS, Kleinman MH, Lake AM, Forman J, Midle JB. Newspaper coverage of suicide and initiation of suicide clusters in teenagers in the USA, 1988-1996: A retrospective, population-based, case-control study. Lancet Psychiatry. 2014; 1(1): 34-43. https://doi.org/10.1016/S2215-0366(14)70225-1

22. Etzersdorfer E, Sonneck G. Preventing suicide by influencing mass-media reporting: The Viennese experience 1980-1996. Archives of Suicide Research. 1998; 4: 67-74. https://doi.org/10.1080/13811119808258290

23. Asociación de Investigación, Prevención e intervención de conductas suicidas. Los medios de comunicación y las conductas suicidas. 2015. Disponible en: https://www.redaipis.org/wp-content/uploads/2015/02/Los-medios-de-comunicacio-n-y-las-conductas-suicidas.pdf

24. Hagihara A, Abe T, Omagari M, Motoi M, Nabeshima Y. The impact of newspaper reporting of hydrogen sulfide suicide on imitative suicide attempts in Japan. Soc Psychiatry Psychiatr Epidemiol. 2014; 49: 221-229. https://doi.org/10.1007/s00127-013-0741-8

25. Cheng Q, Chen F, Yip PSF. Media effects on suicide methods: A case study on Hong Kong 1998-2005. Plos One. 2017; 1-12.

https://doi.org/10.1371/journal.pone.0175580

26. Pérez SA. Los medios de comunicación y el suicidio. Evidencia y sugerencias. S.f. (Consultado el 3 de julio 2021). Disponible en: https://www.redaipis.org/wpcontent/uploads/2015/02/los-medios-de-comunicacin-y-el-suicidio.pdf

27. Ayers JW, Althouse BM, Leas EC, Drezde M, Allem JP. Internet searchers for suicide following the release at 13 reasons why. JAMA Intern Med. 2017; 177(10): 1527-1529. https://doi.org/10.1001/jamainternmed.2017.3333

28. Bridge J, Greenhouse JB, Ruch D, Stevens J, Ackerman J, Sheftall AH, et al. Association Between the release of Netflix's. 13 reasons why and suicide rates in the United States: An interrupted times series analysis. Journal of the American Academy of Child and Adolescent Psychiatry (en prensa). 2019.

https://doi.org/10.1016/j.jaac.2019.04.020

29. Sinyor M, Pirkis J, Picard A, Mckeown D, Vincent M, Cheung M, et al. Towards a shared understanding: Perspectives from Toronto's first media forum for suicide prevention. Canadian Journal of Public Health. 2016; 107(3): 330.

https://doi.org/10.17269/CJPH.107.5437

30. Olson R. Suicide contagion \& suicide clusters. Center of Suicide Prevention. 2013. Disponible en: https://www.suicideinfo.ca/wp-content/uploads/2016/08/ iE10_Web-Version.pdf

31. Chen Y, Liao S, Teng P, Tsai C, Fan H, Lee W, et al. The impact of media reporting of the suicide of a Singer on suicide rates in Taiwan. Soc Psychiatry Psychiatr Epidemiol. 2012; 47: 215-221. https://doi.org/10.1007/s00127-010-0331-y 
Implicaciones bioéticas en el «efecto de contagio» del suicidio

32. Chen Y, Tsai P, Chen P, Fan C, Hung GC, Cheng A. Effect of media reporting of the suicide of a singer in Taiwan: The case of Ivy Li. Soc Psychiat Epidemiol. 2010; 45: 363-369. https://doi.org/10.1007/s00127-009-0075-8

33. Martin HJ, Rennhoff AD. Advertising content and avoidance, new media or old media, and media coverage of celebrity suicides. Journal of Media Economics. 2016; 29(2): 2, 49-50. https://doi.org/10.1080/08997764.2016.1188637

34. Van den Bulck $\mathrm{H}$, Claessens $\mathrm{N}$. Celebrity suicide and the search for the moral high ground: Comparing frames in media and audience discussions of the death of a Flemish celebrity. Critical Studies in Media Communication. 2013; 30(1): 6984. https://doi.org/10.1080/15295036.2011.645496

35. Choi YJ, Oh H. Does media coverage of a celebrity suicide Trigger copycat suicides? Evidence from Korean cases. Journal of Media Economics. 2016; 29(2): 92-105. https://doi.org/10.1080/08997764.2016.1170020

36. Harshe D, Karia S, Harshe S, Shah N, Harshe G, De Sousa A. Celebrity suicide and its effect on further media reporting and portrayal of suicide: An exploratory study. Indian Journal of Psychiatry. 2016; 58(4): 443-447.

https://doi.org/10.4103/0019-5545.196704

37. Stack S. Media impact on suicide: A quantitative review of 293 findings. Social Science Quarterly. 2000; 81(4): 957-971.

38. Schäfer M, Quiring $O$. The press coverage of celebrity suicide and the development of suicide frequencies in Germany. Health Communication. 2015; 30: 11491158. https://doi.org/10.1080/10410236.2014.923273

39. Biddle L, Donovan J, Hawton K, Kapur N, Gunnell D. Suicide and the Internet. BMJ. 2008; 336(7648): 800- 802. https://doi.org/10.1136/bmj.39525.442674.AD

40. Recupero R, Harmss E. Noble JM. Googling suicide: Surfing for suicide information on the internet. J Clin Psychiatry. 2008; 69(6): 878-888.

https://doi.org/10.4088/JCP.v69n0601

41. BMJ. Media influence on suicide. BMJ. 2003; 326: 498.

https://doi.org/10.1136/bmj.326.7387.498

42. The Hindu. Suicide reports in media can affect vulnerable people: study. (Consultado el 19 de julio de 2021). 30 de julio de 2018. Disponible en: https:// www.thehindu.com/sci-tech/health/suicide-reports-in-media-can-affect-vulnerablepeople-study/article24555237.ece\#

43. Hittner JB. How robust is the Werther effect? A re-examination of the suggestion-imitation model of suicide. Morality. 2005; 10(3): 193-200.

https://doi.org/10.1080/13576270500178112

44. Espada A. Periodismo práctico. Madrid: Espasa Calpe; 2008.

45. Ministerio de Sanidad, Política e Igualdad. Guía de práctica clínica de prevención y tratamiento de la conducta suicida. (Consultado el 3 de julio 2021). 2012. Disponible en: https://portal.guiasalud.es/wp-content/uploads/2020/09/gpc_481_ conducta_suicida_avaliat_resum_modif_2020_2.pdf

46. Arnett RC, Harden JM \& Bell LM. Communication ethics literacy. California: Sage; 2009.

Medicina y Ética - Enero-Marzo 2022 - Vol. 33 - Núm. 1 


\section{E. Benítez Camacho}

47. Wilkins L, Christians, CG. The Handbook of mass media ethics. Nueva York: Routledge; 2009. https://doi.org/10.4324/9780203893043

48. Organización Panamericana de la Salud/Organización Mundial de la Salud. Cambiando la cobertura periodística del suicidio en América Latina y el Caribe. 2019. Disponible en: https://www.paho.org/hq/index.php?option=com_content\& view=article\&id=15409:cambiando-la-cobertura-periodistica-del-suicidio-en-america-latina-y-el-caribe\&ltemid $=72543 \&$ lang $=e s$

49. Organización Mundial de la Salud. Prevención del suicidio, un imperativo global. 2014. Disponible en: http://www.who.int/mental_health/suicide-prevention/ world_report_2014/es/

50. Organización Mundial de la Salud. Preventing suicide: A resource for media professionals; update 2017. Disponible en: https://www.who.int/mental_health/suicide-prevention/resource_booklet_2017/en/

51. Carlyle K. The role of social media in promoting understanding of violence as a public health issue. Journal of communication in healthcare. 2017; 10(3): 162164. https://doi.org/10.1080/17538068.2017.1373907

52. Herrera R, Ures MB, Martínez JJ. El tratamiento del suicidio en la prensa española: ¿Efecto Werther o efecto papageno? Rev. Asoc. Esp. Neuropsiq. 2015; 35(125): 123-134. https://doi.org/10.4321/S0211-57352015000100009

53. Skehan J, Maple M, Fisher J, Sharrock G. Suicide bereavement and the media: A qualitative study. Advances in Mental Health. 2013; 11(3): 223-237. https://doi.org/10.5172/jamh.2013.11.3.223

54. Maiorano A, Lasalvia A, Sampogna G, Pocai B, Ruggeri M, Henderson C. Reducing stigma in media professionals: Is there room for improvement? Results from a Systematic Review. The Canadian Journal of Psychiatry. 2017; 62(10): 702-715. https://doi.org/10.1177/0706743717711172

55. McGinty EE, Kennedy-Hendricks A, Choksy S, Barry CL. Trends in news media coverage of mental illness in the United States: 1995-2014. Health Affairs. 2016; 35(6): 1121-1129. https://doi.org/10.1377/hlthaff.2016.0011

56. Fondo de las Naciones Unidas para la Infancia. Comunicación. Infancia, adolescencia. Guía para periodistas. 2017. Disponible en: https://www.unicef.org/argentina/sites/unicef.org.argentina/files/2018-04/COM-5_Suicidio_Interior_WEB.pdf

57. Organización Mundial de la Salud. Prevención del suicidio. Un instrumento para profesionales de los medios de comunicación. Ginebra. 2010. Disponible en: http://www.who.int/mental_health/media/media_spanish.pdf

58. Organización Panamericana de la Salud. Seminario para periodistas: cómo reportar un suicidio. 28 de marzo de 2017. Disponible en: https://www.paho.org/ hq/index.php?option=com_content\&view=article\&id=13069: webinar-for-journalistsbest-practices-for-reporting-on-suicide\&ltemid=41080\&lang $=e s$

59. Organización Panamericana de la Salud/Organización Mundial de la Salud. Cambiando la cobertura periodística del suicidio en América Latina y el Caribe. 9 de septiembre de 2019. Disponible en: https://www.paho.org/hq/index.php?option- 
Implicaciones bioéticas en el «efecto de contagio» del suicidio

=com_content\&view=article\&id=15409:cambiando-la-cobertura-periodistica-delsuicidio-en-america-latina-y-el-caribe\&ltemid=72543\&lang=es

60. Maloney J, Pfuhlmann B, Arensman E, Coffey C, Gusmão R, Poštuvan V, Scheerder G, Sisask M, van der Feltz-Cornelis CM, Hegerl U, Schmidtke A. How to adjust media recommendations on reporting suicidal behavior to new media developments. Arch Suicide Res. 2014; 18(2): 156-69.

https://doi.org/10.1080/13811118.2013.824833

61. Bilbao J, Alcocer A, Lallemand L, Lío JF, Vargas R. Reportes de suicidios en prensa de Barranquilla, un enfoque desde la salud pública. Rev CES Salud Pública. 2016; 7(2): 2-9. https://doi.org/10.21615/cessp.7.2.1

62. Robinson J, Rodrigues M, Fisher S, Bailey E, Herrman H. Social media and suicide prevention: Findings from a stakeholder survey. Shanghai Archives of Psychiatry. 2015; 27(1): 27-35.

63. Infobae. La nota suicida que Kate Spade dejó a su pequeña hija de 13 años. 5 de junio de 2018. Disponible en: https://www.infobae.com/america/eeuu/2018/ 06/05/la-nota-suicida-que-kate-spade-le-dejo-a-su-pequena-hija/

64. Infobae. La hermana de Kate Spade habló tras el suicidio de la diseñadora y provocó un escándalo en la familia. 6 de junio de 2018. Disponible en: https:// www.infobae.com/america/entretenimiento/2018/06/06/la-hermana-de-kate-spadehablo-tras-el-suicidio-de-la-disenadora-y-provoco-un-escandalo-en-la-familia/ 65. Augustyn, A. Anthony Bourdain. Encyclopaedia Britannica. 21 de junio de 2018. Disponible en: https://www.britannica.com/biography/Anthony-Bourdain

66. El País. Anthony Bourdain perdió el $90 \%$ de su fortuna antes de suicidarse. 7 de junio de 2018. Disponible en: https://elpais.com/elpais/2018/07/06/gente/ 1530873478_752828.html

67. Colman I. Responsible reporting to prevent suicide contagion. CAMJ, 2018; 190(3): E898-E899. https://doi.org/10.1503/cmaj.180900

68. Rice S, Robinson J, Bendall S, Hetrick S, Cox G, Bailey, et al. Online and social media suicide prevention interventions for young people: A focus on implementation and moderation. Journal of The Canadian Academy of Child and Adolescent Psichiatry. 2016; 25 (2): 80-86.

69. Morais TCA, Monteiro PS. Los conceptos de vulnerabilidad humana y la Integridad individual para la bioética. Rev. Bioét. 2017; 25(2): 311-319.

https://doi.org/10.1590/1983-80422017252191

70. Feito L. Vulnerabilidad. An. Sist. Sanit. Navarr. 2007; 30(Supl. 3): 7-22.

https://doi.org/10.4321/S1137-66272007000600002

71. Páez R. La vulnerabilidad social en la bioética. Revista Iberoamericana. 2017; 5: 1-14. https://doi.org/10.14422/rib.i05.y2017.001

72. Sgreccia E. Manual de Bioética I. Fundamentos y ética biomédica. Madrid: Biblioteca de Autores Cristianos; 2009.

73. Nyberg T, Myrberg I, Omerov P, Steineck G, Nyberg U. Depression among parents two to six years following the loss of a child by suicide: A novel prediction model. Plos One. 2016; 11(10): e0164091.

https://doi.org/10.1371/journal.pone.0164091

Medicina y Ética - Enero-Marzo 2022 - Vol. 33 - Núm. 1 


\section{E. Benítez Camacho}

74. Spillane A, Larkin C, Corcoran P, Matvienko-Sikar K, Arensman E. What are the physical and psychological health effects of suicide bereavement on family members? Protocol for an observational and interview mixed-methods study in Ireland. BMJ Open. 2017; 7(3): 8. https://doi.org/10.1136/bmjopen-2016-014707

75. García JJ. Bioética personalista y bioética principialista. Perspectivas. Cuadernos de Bioética. 2013; XXIV(1): 67-76.

Esta obra está bajo licencia internacional Creative Commons Reconocimiento-NoComercial-Compartirlgual 4.0.

\section{(c) (i) (2)(2)}

BY NG SA 